\title{
THE DESIGN AND ASSESSMENT OF ATTENTION-GETTING REAR BRAKE LIGHT SIGNALS
}

\author{
M. Lucas Neurauter, Robert E. Llaneras \& Walter W. Wierwille \\ Virginia Tech Transportation Institute \\ Blacksburg, Virginia, USA \\ Email: $\underline{\text { lneurauter@,vtti.vt.edu }}$
}

\begin{abstract}
Summary: This paper summarizes work intended to further characterize and develop rear brake light signals likely to improve driver reaction to hard-braking lead-vehicle events, emphasizing unique and novel approaches not previously studied. The work developed optimized signal lighting configurations, including specifications for LED signal approaches (flash frequencies, brightness levels, patterns), and performed field evaluations to assess eye-drawing capability of candidate signals for drivers who were looking away from the forward view. Results indicate that newer rear signaling designs can be very effective at drawing drivers' eyes back to the forward roadway, and that flashing and brightness are two important signal properties moderating effectiveness (attention-getting). Remarkable performance gains can be achieved via use of LED signal approaches which both flash and increase signal intensity or lamp brightness.
\end{abstract}

\section{INTRODUCTION}

Crash database studies have shown that approximately 29 percent of all crashes are rear-end crashes, a figure that has remained steady during the past decade (NTSB, 2001; NHTSA, 2007). These crashes often result in serious injuries, loss of productive time, and high levels of property damage, particularly vehicle damage. Furthermore, these crashes often cause traffic congestion, resulting in reduced highway throughput. Data suggest that failure to respond (or delays in responding) to a stopped or decelerating lead vehicle is generally a result of distraction and, in particular, improper allocation of visual attention (Lee, Llaneras, Klauer, and Sudweeks, 2007).

The work described here is part of a larger program of research intended to evaluate rear signaling applications designed to reduce the frequency and severity of rear-end crashes (Wierwille, Llaneras, and Neurauter, 2008). This approach to the rear-end crash problem is based on the premise that a successful rear signaling system would work to redirect driver visual glance and attention to the forward roadway (for cases involving a distracted driver), as well as improve the driver's ability to discern hard-braking events by increasing the saliency or meaningfulness of the brake signal (for drivers already looking forward).

This particular effort was directed at quantifying the attention-getting capability and discomfort glare of a set of candidate test configurations using driver judgments, as well as eye-drawing metrics of a subset of configurations using an uninformed detection event methodology. Candidate test configurations included proposed approaches from automotive companies, configurations based on expert opinions, and the Incandescent Traffic Clearing Lamp (TCL) found to provide the best overall attention getting performance in earlier studies conducted at the Virginia Tech Transportation Institute (Wierwille, Lee, and DeHart, 2003, 2005). 


\section{METHOD}

Testing was performed with a group of 80 naïve drivers (no previous exposure to the lighting arrays) under static conditions where participants were not required to formally drive. Initial uninformed lighting event detection trials (administered before drivers were informed about the true purpose of the study) were used to assess eye-drawing capability for a subset of the lighting configurations. The uninformed event trials were intended to supplement subjective ratings with performance-based values (i.e., incidence of glances to the forward view when otherwise occupied).

\section{Instrumentation and Test Apparatus}

Assessments were performed using a vehicle mock-up test apparatus (see close-up within Figure 1) comprised of a rigid composite metal backing with an attached vehicle appliqué matched to average dimensions found on typical passenger vehicles. The mock-up housed working brake lamp units. To achieve relatively high brightness using light-emitting diode (LED) units, individual units were used in multiples (gangs), in numbers of six for each outboard 'lamp' and three for the center high-mounted stop lamp (CHMSL). The high output luminance of the lamps was configured to correspond with output levels of the TCL found effective in previous work. Programming was incorporated which allowed experimenters to vary the brightness, flash patterns, and flash frequencies of the lamps.

The vehicle that the participants sat in was instrumented with a data acquisition system that allowed for capture of time-synchronized video of the participant's (driver's) eye position and the state of the lead vehicle's brake lamps. This video allowed for post hoc analysis of eyeglance behavior relating to when participants redirected their glance (if that occurred) to the lights and corresponding latencies.

\section{Procedure}

Participants were led to believe the purpose of the study was to evaluate an in-vehicle navigation system, with eye-drawing (signal attention-getting) assessed during a series of uninformed trials. During these uninformed trials, participants (seated in the driver's seat) were asked to complete in-vehicle tasks using an in-car navigation system, causing them to direct their gaze away from the forward roadway. The display and controls for the navigation system were located along the vehicle centerline, to the right of the steering wheel, near the top of the instrument panel (refer to Figure 1). The vehicle mock-up with the candidate brake signals was positioned $30.5 \mathrm{~m} \mathrm{(100} \mathrm{ft}$ nominal eye distance) straight ahead of the subject vehicle (again, all work was performed with the cars stationary). Lights on the mock-up were activated when the participant was glancing toward the navigation system display. This was accomplished through the use of handheld radios. The in-vehicle experimenter would inconspicuously signal the experimenter operating the vehicle mock-up when the participant was looking at the navigation system. 


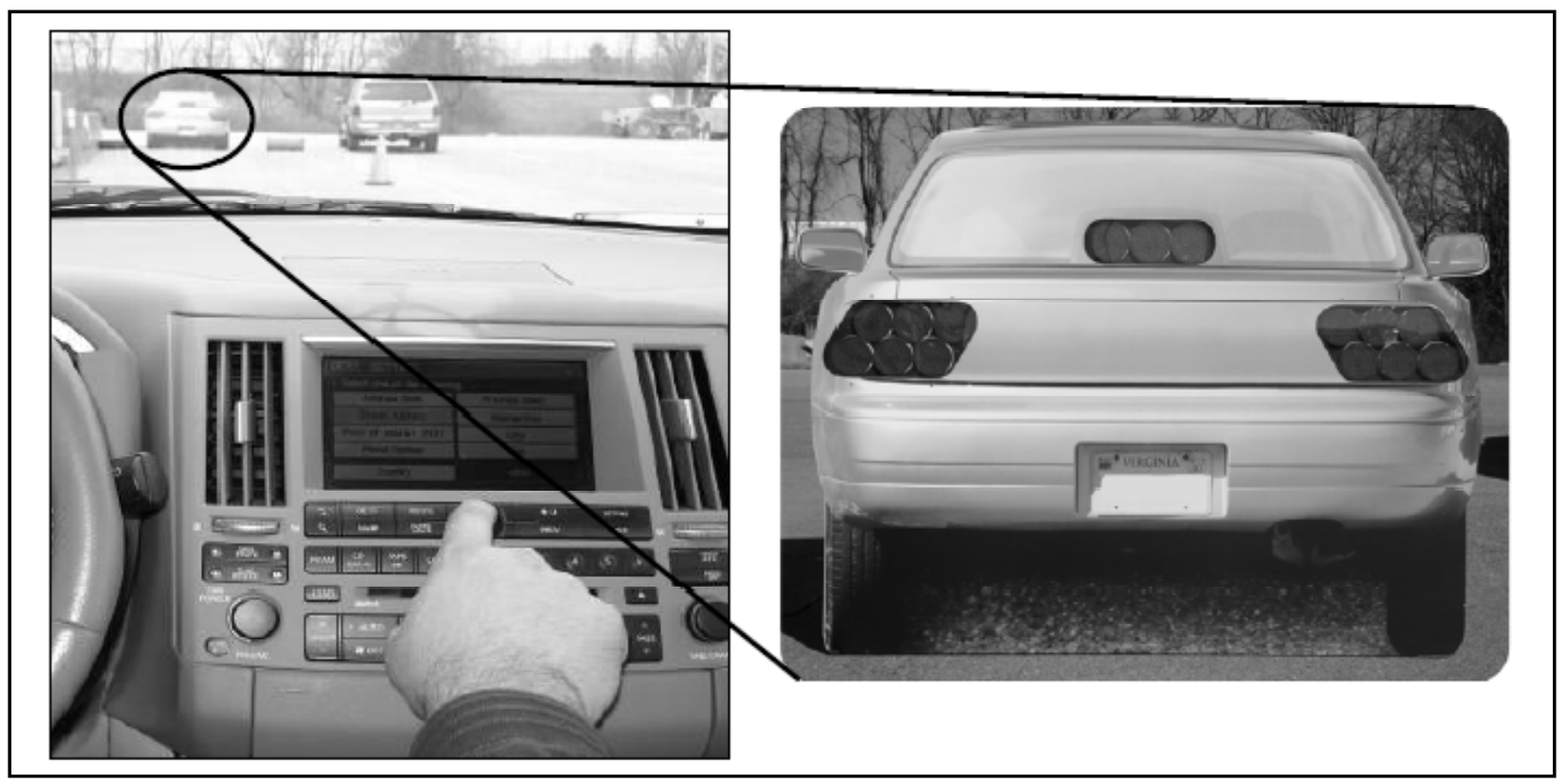

Figure 1. Experimental set-up. Driver's perspective showing the navigation system and the external view of the rear signaling mock-up (with enlarged close-up view of the mock-up with working brake lamps)

In all, there were three triggering events for each participant, all of which occurred without informing the participant. These triggering events occurred as follows: once while receiving instruction but while the participant was looking at the navigation system display; once when selecting among menu items in the navigation system; and once during text entry into the navigation system. These three events were chosen to reflect increasing levels of visual, cognitive, and manual loading. The number of occurrences of eye-drawing (participants looking forward to the mock-up) and the times it took them to re-direct their gaze forward were measured and served as key dependent measures for assessing eye-drawing capability. Although participants did not drive the vehicle during the navigation task elements (and therefore had no need to look forward), the hypothesis was that effective signals would compel individuals to redirect their gaze forward. In other words, the eye-drawing capability of some of the signals would cause the driver to look forward even though the need to look forward (as if driving) was not present. Measures of eye-drawing capability (i.e., whether or not drivers looked forward in response to the signal activations and latency of looking forward) were used in conjunction with the subjective evaluations of the entire set of available lighting configurations (obtained later) to narrow the pool of candidate signals. These subjective evaluations provided ratings of attentiongetting and discomfort glare from both near and far following distances, and also from an adjacent lane position.

\section{Rear Lighting Signal Configurations}

Five different rear lighting configurations were tested using the uninformed event detection paradigm. Each configuration was presented for a duration of 5 seconds, preceded by a 1-second presentation of baseline (normal-level brake lighting), for a total presentation of 6 seconds. Eighty drivers participated in the uninformed event detection paradigm. This number was considered to be large, but was necessary because of the between-subjects design. There were 16 
drivers per display condition. The use of a between-subjects design was considered necessary because re-exposure, after informing participants of the ruse, would not have provided the surprise (uninformed event) effect that was a goal of the experiment. As mentioned previously, following this ruse, a subset of participants was asked to provide subsequent attention-getting and discomfort glare ratings of these candidate lighting patterns (including an additional four patterns not listed here).

- Incandescent TCL: Incandescent electro-mechanical unit used for CHMSL and combined with steady burn LED outboard lamps at increased brightness. This unit was carried over from previous testing.

- Simultaneous Flashing (Increased Brightness): LED lamps optimized in frequency according to previous experiments. All lamps flashed at $5.0 \mathrm{~Hz}$ with full output brightness level.

- Simultaneous Flashing (No Increase): LED lamps optimized in frequency according to previous experiments. All lamps flashed at $5.0 \mathrm{~Hz}$ with normal illumination level (corresponding with Baseline level).

- Outboard Simultaneously Flash, CHMSL Alternate: LED lamps optimized in frequency according to previous experiments. CHMSL flashed alternatively with outboard lamps at $4.75 \mathrm{~Hz}$ with full output brightness level.

- Baseline: LED Implementation of conventional, steady-burn brake lamps. All LED lamps (including outboard units and CHMSL with three LED units) steady-burn at a normal illumination level.

\section{RESULTS}

\section{Eye-Drawing}

Results generally support the 'Simultaneous Flashing (Increased Brightness)' configuration as most effective in drawing the participants eyes back to the forward view. A remarkable $69 \%$ of participants noticed the lighting onset on at least one occasion when simultaneous flashing and increased brightness were combined (Figure 2). Subsequent video eye-glance analysis confirmed that drivers did in fact look up in response to the signal. This finding marked a significant improvement above all configurations aside from the 'Outboard Simultaneous Flash, CHMSL Alternate' (plotted results with a common letter do not differ significantly at the $\alpha=0.05$ level using a chi-square test). None of the participants under the Baseline configuration noticed the lighting, suggesting that current rear lighting is much less effective in drawing the participants eyes back to the forward view. It is important to note that these results are for the case in which bright daylight floods the lead vehicle and the participant is involved in an in-car task requiring visual and cognitive load, a task that could be considered typical of more complex in-car tasks. Flashing alone (without increased brightness) demonstrated a modest improvement in eye drawing; however, this configuration is far less effective than flashing with increased brightness insofar as the testing performed in the current study is concerned. Although the below figure charts the percentage of participants who looked forward at the mock-up during any of the three separate exposures, it is also important to note that under light load (first exposure) for the 'Simultaneous Flashing (Increased Brightness)' configuration, participants looked up to the rear lighting display $56 \%$ of the time while glancing to the navigation display. 


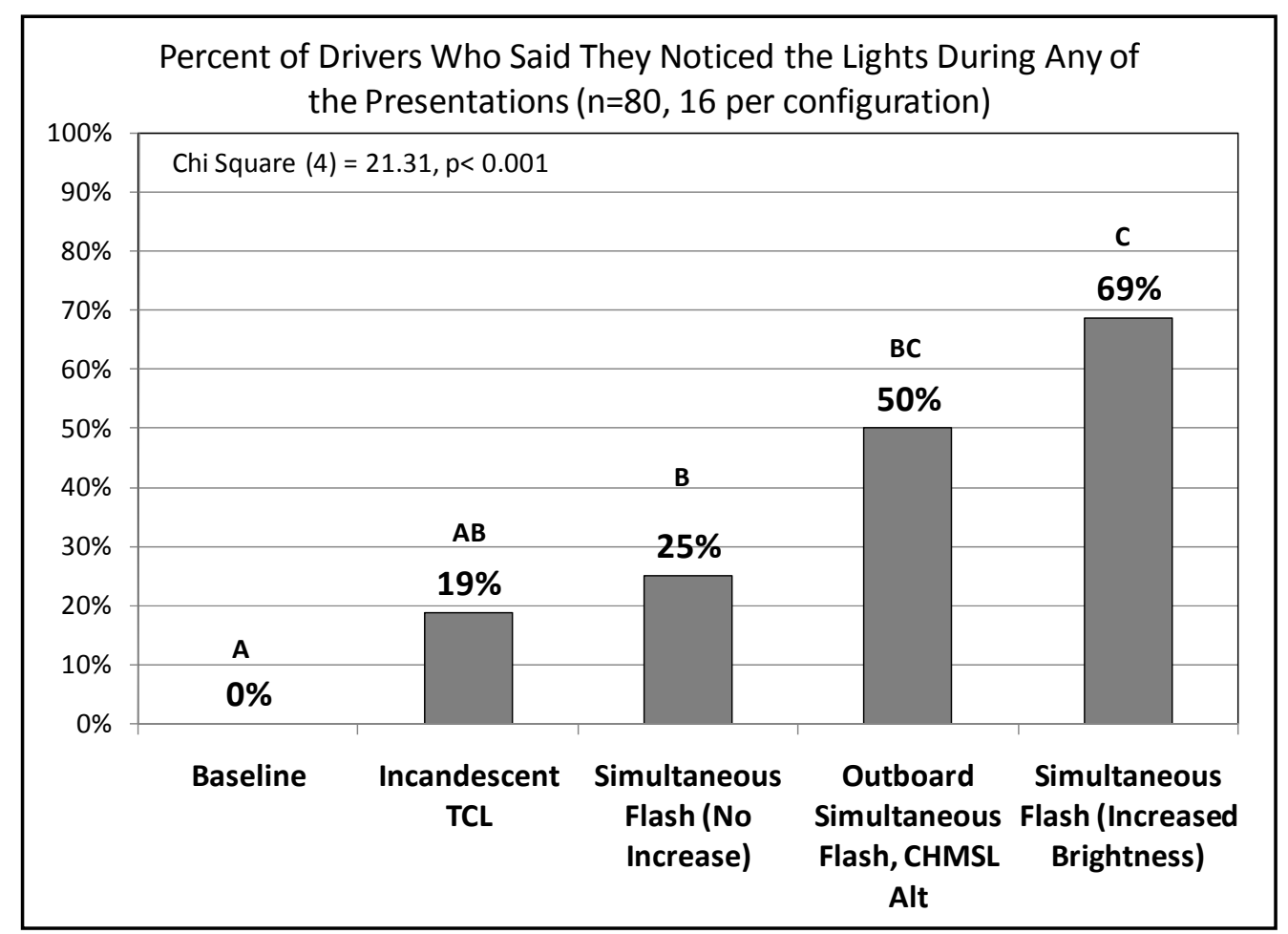

Figure 2. Percentages of participants who looked up in response to the rear signal lamp activations (derived from video recordings)

Latency data for the first exposure were analyzed to determine whether some signals solicited faster eye-drawing than others during the 6-second exposure interval. The data were analyzed by means of a one-way between-subjects analysis-of-variance. Results were significant with $\mathrm{F}(4,79)$ $=3.18, p<0.01$. The plot shows that the Baseline configuration did not cause participants to look up at all; that is, all response times were set at 6 seconds. Only the 'Outboard Simultaneous Flash with CHMSL Alternate' and the 'Simultaneous Flash (Increased Brightness)' were significantly superior in engendering quicker eye-drawing time. Both of these configurations used the increased (emergency) brightness level for the lighting exposure. Note that the Incandescent TCL and the 'Simultaneous Flash (No Increase)' configurations had some eyedrawing capability, but not to the extent that they were signficantly different from Baseline. 


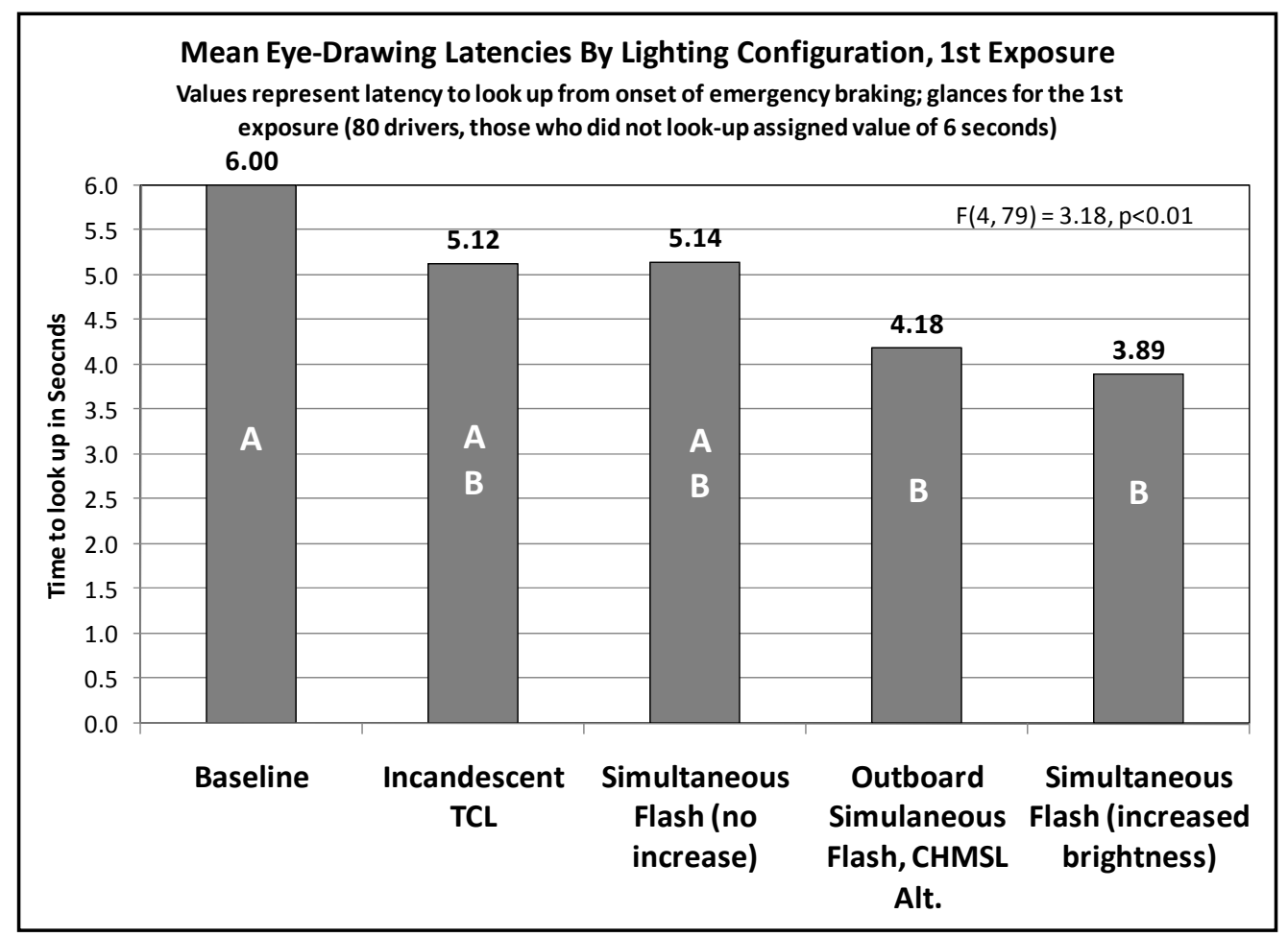

Figure 3. Mean look-up response times on first exposure to the lighting display as a function of lighting configuration

\section{Subjective Ratings of Attention Getting}

In terms of rated attention getting, whether looking straight ahead at a nominal eye-distance of $30.5 \mathrm{~m}$ (100 ft) or looking to the right at an angle of 30 degrees, driver ratings corresponded highly with findings from the uninformed event-detection paradigm. Clearly, flashing and increased brightness play a major role in attaining high ratings by the research participants. These results suggest that both flashing and brightness are effective in increasing the attentiongetting ratings.

\section{SUMMARY \& CONCLUSIONS}

Results of the post hoc tests indicate that all three LED signal configurations led to improved eye-drawing capability over the conventional (baseline) braking signal, and that the simultaneous flashing with increased brightness demonstrated superior performance relative to the other configurations with the exception of the 'Outboard Simultaneously Flashing, CHMSL Alternate' configuration. Of particular interest is that while simultaneous flashing of brake lamps without an increase in brightness led to significant improvement over baseline, pairing the flashing with increased brightness significantly enhanced the eye-drawing capability, nearly tripling it compared with flashing alone. A remarkable $69 \%$ of participants noticed the lighting on at least one occasion during presentations of the 'Simultaneous Flashing (Increased Brightness)' configuration, compared to $0 \%$ for the Baseline configuration. 
Both look-up (eye drawing) data and interview data support the hypothesis that simultaneous flashing of all rear lighting, combined with increased brightness, would be effective in redirecting the driver's eyes to the lead vehicle when the driver is looking away with tasks that involve visual load.

\section{ACKNOWLEDGMENTS}

This research was sponsored by the National Highway Traffic Safety Administration under Contract DTNH22-05-D-01019.

\section{REFERENCES}

Lee, S., Llaneras, E., Klauer, S., and Sudweeks, J. (2007). Analysis of rear-end crashes and near-crashes in the 100-Car naturalistic driving study to support rear-signaling countermeasure development (No. DOT HS 810 846). Washington, D.C.: National Highway Traffic Safety Administration.

National Highway Traffic Safety Administration (2007). Traffic safety facts 2005: A compilation of motor vehicle crash data from the Fatality Analysis Reporting System and the General Estimates System (No. DOT HS 810 631). Washington, D.C.: U.S. Department of Transportation.

National Transportation Safety Board (2001). Special investigation report: Vehicle- and infrastructure-based technology for the prevention of rear-end collisions (NTSB/SIR-01/01). Washington, D.C.

Wierwille, W., Lee, S., and DeHart, M. (2005). Task 3 report: Test road evaluation of enhanced signals (No. DOT HS 809 864). Washington, D.C.: National Highway Traffic Safety Administration (NHTSA).

Wierwille, W., Lee, S., and DeHart, M. (2003). Task 2 report: Identification of potential signal enhancements (No. DOT HS 809 597). Washington, D.C.: National Highway Traffic Safety Administration (NHTSA).

Wierwille, W., Llaneras, R., and Neurauter, M. (2008). Evaluation of enhanced brake lights using surrogate safety metrics: Task 1 report: Further characterization and development of rear brake light signals (NHTSA Contract Number DTNH22-05-01019 Task Order 14). Blacksburg, VA: Virginia Tech Transportation Institute. 